roulées en tire-bouchon. A Aïn M'lila nous constatâmes, M. Trabut et moi, la constance de ce caractère sur des milliers de pieds. M. Pomel nous a dit depuis avoir souvent rencontré cette plante dans les HautsPlateaux, mais ne l'avoir jamais vue en fleur. Quelques pieds rapportés d'Aïn M'lila ayant fleuri à Alger nous permettront de la décrire.

L'A. tortifolium appartient au type de l'A. Ampeloprasum L. Il en diffère par ses feuilles peu carénées à nervure arrondie, lisses, à peine papilleuses à la loupe, fortement enroulées en spires contiguës ou imbriquées de manière à former de véritables cornets; par les pièces du périanthe plus courtes, peu carénées, à peine papilleuses. Cette plante pousse en touffes et a de nombreux bulbilles portés sur des pédoncules robustes.

Il forme, avec l'A. Duricanum de Bône et l'A. getulum du Mzi, une série de petites espèces gravitant autour de l'A. Ampeloprasum. L'A. Pardoi de Sétif nous parait constituer un type bien distinct.

Enfin nous mentionnerons une forme à gros fruits de l'Helianthemum Lippii trouvée à la Montagne de sable; une variété très grandiflore du Linaria reflexa, ce protée végétal, trouvée au Tougourt avec le Mentha candicans, qui paraît assez répandu dans l'Aurès, M. Trabut l'ayant depuis rapporté du Chéliah, ainsi qu'un de ses hybrides avec le $\boldsymbol{M}$. rotundifolia. Il convient également de noter l'Argyrolobium Saharæe que l'on n'avait encore trouvé qu'au Mzab.

HERBORISATION DANS LE MASSIF DE L'AURES, LES 10, 11, 12, 13, 14 JUILLET, par 11. I. TRABUT.

Au retour de la session de Biskra, à la fin d'avril, un arrêt de quelques jours à Batna a permis aux membres de la Société botanique de faire deux excursions dans la région montagneuse de l'Aurès; l'une à Lambèse, l'autre au djebel Tougourt; mais il ne s'agissait que de faire connaissance avec le facies de la végétation arborescente et avec la flore du printemps, la grande majorité des plantes étaient encore bien loin de la floraison.

C'est en juin qu'il faut explorer ces massifs montagneux, dont les cimes dépassent fréquemment 2000 mètres. Retenu à Alger par des occupations professionnelles, ce n'est que le 10 juillet que j'abordai de nouveau l'Aurès, ayant pour compagnon de voyage M. Fischeur, professeur de géologie; notre but était d'explorer les sommets et les parties supérieures des vallées en nous maintenant à une altitude de 1500 à 2300 mètres, zone où règne, en cette saison, une température agréable. En quittant Lambèse par la route de Bordj Taza, on traverse la zone 
forestière des taillis de Chênes verts (Quercus Ilex); le sol est formé par des alternances de marnes et de grès du Cénomanien. Nous notons :

Quercus Ilex

Juniperus 0xycedrus.

Rhamnus Alaternus.

Erinacea pungens.

Genista microcephala.

Acanthyllis tragacanthoides.

Santolina squarrosa.

Rosmarinus officinalis.

Thymus ciliatus.

Sideritis atlantica.

Phlomis Herba-venti.

Teucrium Polium.

Helianthemum rubellum.

Ruta angustifolia.

Anthemis tuberculata.

Bupleurum oligactis.

Euphorbia luteola (très abondant dans tout le massif de l'Aurès jusqu'aux sommets).

\author{
Asphodelina lutea. \\ Piptatherum paradoxum. \\ Gypsophila compressa. \\ Elæoselinum Fontanesii. \\ Othonna cheirifolia. \\ Echinops spinosus. \\ Atractylis cespitosa. \\ Alkanna tinctoria. \\ Centaurea tougourensis. \\ Artemisia campestris. \\ Phalaris truncata. \\ Stipa gigantea. \\ Trisetum flavescens. \\ Avena bromoides. \\ Poa bulbosa. \\ Melica ciliata. \\ Kœeleria valesiaca. \\ Lolium perenne. \\ Festuca rubra.
}

A Bordj Taza (1400 mètres), l'eau est abondante dans les bas-fonds où dominent le Cirsium monspessulanum, le Senecio giganteus, Festuca arundinacea, Juncus paniculatus, Agrostis alba, Mentha candicans, qui, dans toute la partie élevée du massif de l'Aurès, se substitue au Mentha rotundifolia des stations plus basses; au contact de ces deux espèces (à Lambèse), nous avons trouvé un hybride, $M$. silvestri $\times$ rotundifolia. Un verger avec les arbres fruitiers de l'Europe est en pleine prospérité, les Noyers surtout sont vigoureux; ici, comme dans toutes les vallées élevées du massif de l'Aurès, cet arbre paraît dans un milieu qui lui est éminemment favorable.

Chez le cheik qui nous reçoit sous quelques Figuiers, déjà à leur limite altitudinale, nous pouvons examiner les moissons et commencer une collection intéressante des races de Blés durs cultivés par les indigènes de l'Aurès. Quelques-unes de ces races sont peu connues et fort belles; elles ont dû être introduites et propagées pendant l'occupation romaine.

De Bordj Faga à Medina, nous entrons dans la zone du très curieux Fraxinus dimorpha, le Frêne épineux de l'Aurès, Touzzalt en chaouia. Cet arbre d'une taille moyenne est généralement touffu et plus ou moins taillé en têtard par les indigènes qui coupent les branches à l'automne pour en faire manger les feuilles au bétail. Le tronc est entouré, à la base, d'un fourré épais de pousses broutées à petites feuilles vert foncé d'un aspect très particulier, rappelant les Oléastres broutés et venus 
dans les mêmes conditions. Les fruits de ce Frêne disposés en ombelles persistent parfois d'une année à l'autre ; les fruits de l'année n'étant pas encore mûrs, nous faisons une récolte de ceux de 1891, qui paraissent en fort bon état.

Cette essence encore peu connue devrait ètre propagée en Europe, non seulement dans les bosquets et parcs, mais aussi dans les régions sèches du Midi oú elle pourrait rendre des services comme plante fourragère.

Le Frêne de l'Aurès ne parait pas redouter la sécheresse, et de plus il s'élève dans son pays à 2000 mètres, altitude à laquelle correspond un climat déjà rigoureux.

Le chemin de Médina que nous suivons gravit des escarpements, puis débouche sur un plateau à environ 1600 mètres; les moissons y sont magnifiques et nous notons au passage les plantes dominantes qui sont toutes fortement armées pour résister à la dent des herbivores :

Delphinium orientale.

Euphorbia luteola.

Picnomon Acarna.

Scolymus hispanicus.

Eryngium triquetrum.

- campestre.

Cynara Carduncellus.
Centaurea acaulis.
Mentha Pulegium.
Othonna cheirifolia.
Festuca arundinacea.
Alopecurus pratensis.

Médina ne constitue pas un village, mais un centre de culture important avec quelques habitations dispersées de Chaouias et des tentes de Sahariens venus pour passer l'été. Notre tente est dressée sur la terrasse mème d'une maison entourée de Berberis hispanica, à la lisière d'un bois de Quercus Ilex et de Fraxinus dimorpha.

C'est de ce point que nous partons le lendemain pour faire l'ascension du djebel Chéliah. La plaine élevée de Médina nous offre comme plantes à noter : le Triticum hordeaceum, qui nous a toujours paru l'indice d'excellentes terres à blé; Hordeum secalinum, assez rare en Algérie; Agilops ventricosa, Bromus tectorum; Phalaris truncata, très répandu dans toutes les plaines de la province de Constantine; Juncus glaucus, Passerina virescens, Plantago albicans, Anchusa italica, Centaurea acaulis, Cirsium echinatum, Silybum eburneum, Turgenia latifolia, Medicago sativa, Delphinium orientale, Phlomis Herba-venti, Onopordon macracanthum, Carduus macrocephalus, Picnomon Acarna, Galium verum, Eryngium campestre, Ruta montana, etc.

Nous commençons l'ascension du djebel Chéliah par un ravin du flanc nord où coule l'oued el Hammam. Le sol est toujours formé par des alternances de marnes et de grès ou calcaire gréseux; il est recouvert de boisements assez importants de Chênes verts, de Fraxinus dimor- 
pha, d'Oxycèdres. Mais il est facile de constater que tous ces arbres sont séculaires, on ne trouve pas un sujet de semis et même pas un rejet sur souche; tout est brouté à outrance. L'aspect dépérissant de ces bois était encore plus sensible cette année, les chenilles du Liparis dispar n'ayant pas laissé une feuille sur les Chênes.

Après avoir pris quelques photographies de ces boisements en voie d'extinction rapide, nous ne tardons pas à atteindre la crête d'un contrefort qui nous conduit facilement sur le dòme principal du Chélia. La flore a pris le caractère atlantique, nous cueillons :

Alyssum atlanticum.

Draba hispanica.

lberis Pruitii.

Aethionema saxatile. Arenaria grandiflora. Cerastium Boissieri.

Paronychia aurasiaca.

Sedum acre.

- amplexicaule.

Seseli montanum.

- atlanticum.

Bupleurum spinosum.

Scabiosa crenata.

Jurinea humilis.

Evax Heldreichii.

Inula montana.

Helichrysum lacteum.

Senecio Absinthium.

Catananche cærulea.

Scorzonera pygmæa.

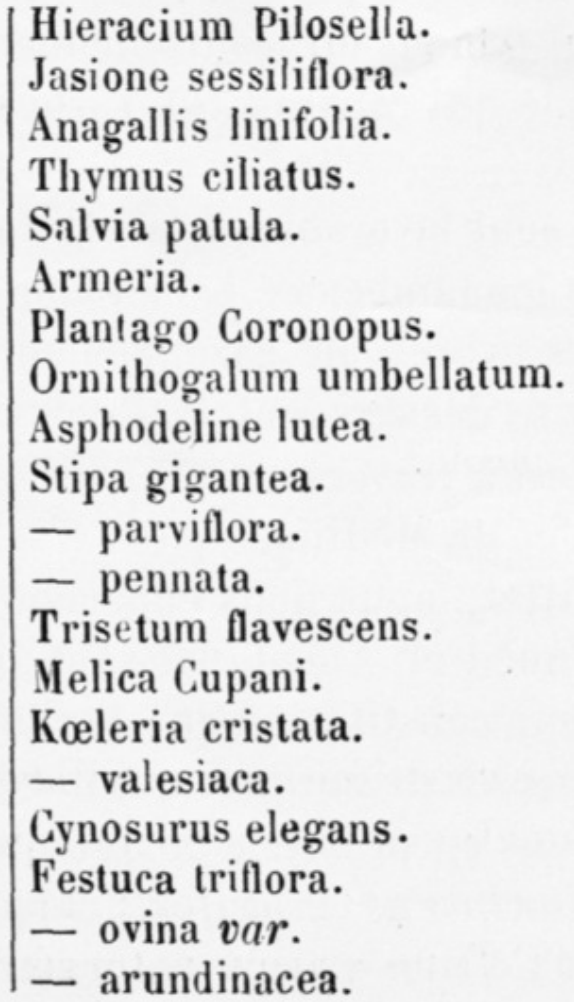

Enfin nous abordons la ceinture de Cèdres. Après avoir un moment admiré la silhouette de ces majestueux'végétaux se détachant en teinte sombre sur le fond blanchâtre des crêtes dénudées, nous pénétrons dans une forêt ruinée; tous ces arbres sont de respectables débris qui peu à peu tombent sans laisser de postérité, tout ce qui est à la portée de l'indigène ou de ses bètes est mutilé ou rongé. De grands lambeaux d'écorce sont enlevés à presque tous les troncs. Quelques sujets rabougris sont tondus rez de terre; de rares Ifs sont réduits à un trone couvert d'une sorte de gazonnement formé par les pousses de l'année qui n'ont pas encore été broutées. Le Juniperus communis var. nana couché sur le sol est aussi passé à l'état de gazon.

Sur les Cèdres, les cônes sont rares; cependant nous observons quelques germinations de l'année, mais pas un pied de deux ans; les jeunes semis seront certainement broutés avant la fin de l'été. Au-dessus de la 
zone des Cèdres, nous retrouvons un grès qui est très favorable à un gazonnement par le Festuca ovina var. el par d'énormes touffes de $S a$ rothamnus Balansa. Enfin, au sommet, nous cueillons : Potentilla pensylvanica et Lamium longiflorum.

Du sommet du Chéliah, point le plus élevé des montagnes de l'Algérie (2331 mètres), nous pouvons observer des relations très intéressantes entre la constitution du sol et la nature de la végétation. Sur les marnes du Cénomanien, qui se délitent facilement, nous apercevons au loin, vers le nord, d'importants boisements. Les calcaires du Turonien, au contraire, se montrent partout stériles et forment, au-dessus de la vallée de l'oued el Abiod, un couronnement blanc d'une grande aridité; la terre végétale qui couvrait ces roches a été depuis longtemps entraînée par les eaux.

Les grès sont favorables à la végétation arborescente, c'est sur eux que sont établis les lambeaux des anciennes forèts de Cèdres; après la destruction des arbres un gazonnement serré s'établit, surtout dans les parties qui se désagrègent facilement, et les sources y sont très fréquentes. C'est à travers ces prairies que nous descendons du versant sud dans la vallée de Médina.

Le 12 juillet, nous nous engageons dans la partie supérieure de la vallée de l'oued el Abiod, laissant le djebel Ichemoul qui nous paraît avoir la même constitution que le Chéliah. A peine avions-nous gravi les premières pentes de collines qui bordent la vallée de Médina, que nous nous trouvions en présence de quelques pieds dispersés, mais de grande taille, du Juniperus thurifera, appelé par les indigènes de ce point Tazenzena. Ce Genévrier a dûformer des peuplements importants dans la partie centrale du massif de l'Aurès; il est aujourd'hui représenté par des sujets de grande taille à cime arrondie, souvent mélangés au Juniperus phænicea, qui s'en distingue facilement de loin par un ton roussâtre.

A partir de 1600 mètres, sur la croupe dénudée du djebel Mahmel, le Juniperus thurifera est assez abondant ainsi que sur le versant N.-0. de ce chaînon dans la vallée de l'oued Abdi jusqu'à Baali oủ il porte le nom chaouia de Aïoual; nous le retrouverons encore dans les forêts de l’État à Sgag.

La partie supérieure de la vallée de l'oued el Abiod est encore boisée; le Pin d'Alep, l'Oxycèdre, le Chêne vert, le Frêne dimorphe, le Dyss (Ampelodesmos tenax), le Bupleurum spinosum, l'Othonna cheirifolia dominent. Vers 1500 mètres la flore change assez brusquement; les plantes caractéristiques et dominantes sont : Pistacia atlantica, Rhamnus lycioides, Artemisia Herba-alba, Thymus ciliatus, Acanthyllis armata, Stipa tenacissima. Dans les jardins, placés sur les 
bords de l'oued comme de véritables oasis, apparaissent l'Abricotier, puis le Noyer et la Vigne; à Anis un énorme Olivier greffé seul dans la région était encore en fleur le 13 juillet; ce témoin d'anciennes cultures doit être contemporain de l'occupation romaine dont on trouve les vestiges à chaque pas.

En descendanț la vallée de l'oued el Abiod du Chéliah jusqu'à Mechounech, on traverserait rapidement toutes les zones de végétations étagées sur les flancs de l'Aurès; qui se succèdent dans l'ordre suivant :

$1^{\circ}$ Prairies à Festuca ovina, bois de Cèdres, avec Taxus baccata et Juniperus nana (pâturages).

$2^{\circ}$ Fraxinus dimorpha, Quercus Ilex, Berberis hispanica, Juniperus thurifera (Blé, Maïs, Pomme de terre).

$3^{\circ}$ Pinus alepensis, Juniperus phœnicea, Juniperus Oxycedrus (mêmes cultures).

$4^{\circ}$ Pistacia atlantica, Rosmarinus officinalis, Artemisia Herbaalba, Nerium Oleander, Ulmus campestris, Celtis australis, Stipa tenacissima (Noyer, Abricotier, Pêcher, Vigne, Grenadier).

$5^{\circ}$ Olivier.

$6^{\circ}$ Dattier.

Nous campons à Baali, joli village chaouia, sur l'oued Abdi, oủ nous retrouvons chez les habitants de nombreux individus ayant conservé le type romain. Le 13, nous explorons la partie supérieure de la vallée jusqu'à Téniet el Abiod. Nous retrouvons dans cette vallée un air de prospérité, une culture plus intensive; dans tous les terrains un peu plans, on voit un champ de Blé entouré de murs en pierres sèches. Les jardins se succèdent le long de la rivière, ce sont les Noyers qui dominent partout; une halte à Haïdous nous permet de noter :

Euphorbia luteola (toujours CCC.). Callipeltis cucullaria.

Artemisia campestris.

- Herba-alba.

Othonna cheirifolia.

Atractylis cespitosa.
Leontodon helminthioides.

Echium italicum.

Thymus algeriensis.

Ziziphora hispanica

Teucrium Polium.

A Téniet el Abiod nous changeons de direction; quittant la vallée de l'oued Abdi, nous nous dirigeons sur Bouzina en franchissant un col du djebel Groumbt-el-Dib, qui sépare la vallée de l'oued Abdi supérieur de la vallée de l'oued Bouzina. Les cultures de céréales s'étendent très haut; à la base de rochers culminants, dans un cailloutis calcaire, nous trouvons encore des moissons d'assez helle venue. C'est la race de Blé appelée dans le pays Hadjini qui domine; les épis sont courts et très aplatis, le grain est glacé, corné, très lourd. L'eau des sources accumulées dans des 
bassins arrose des jardins. Sur les sommets dominent: Prunus prostrata, Rhamnus prostratus, Bupleurum spinosum, Othonna cheirifolia, Evax Heldreichi, Carduncellus pinnatus, Helichrysum lacteum, Hieracium Pilosella, Calamintha alpina, Plantago Coronopus CCC, Euphorbia luteola, Erodium trichomancefolium, Festuca ovina, Triticum hordeaceum, Genista ramosissima, G. microcephala, Daphne Gnidium. A Bouzina, village très pittoresque sur une magnifique source, dans un lit encaissé de torrent, nous prenons un peu de repos avant de faire l'ascension du djebel Mahmel, d'où nous gagnons la maison forestière de Sgag. Le djebel Mahmel court à peu près dans la même direction que le djebel Groumt-el-Dib, les chaînes des Beni Daoud, des Beni Sliman et de l'Ahmarkadou, qui, partant de Médina, vont en se dirigeant comme les doigts d'une main tournée vers Biskra. Le point culminant du djebel Mahmel atteint 2300 mètres; toute cette longue crête est formée de calcaire parfois creusé de grottes, la terre végétale a disparu et la végétation arborescente est réduite à des vestiges de Juniperus thurifera très anciens et dépérissants. Nous retrouvons ici une grande partie des plantes du Chéliah et nous notons encore:

Erysimum grandiflorum.

Aethionema saxatile.

Sinapis pubescens.

Alsine setacea.

Erodium montanum.

Sarothamnus Balansæ.

Prunus prostrata.

Paronychia aurasiaca.

Sedum acre.

Bupleurum spinosum.

Seseli montanum.

Othonna cheirifolia.

Jurinea humilis .

Evax Heldreichii.

Inula montana.

Anthemis tuberculata.

Helichrysum lacteum.
Hieracium Pilosella.

Androsace maxima.

Linaria heterophylla.

Calamintha alpina.

Armeria allioides.

Plantago Coronopus.

Euphorbia Juteola.

Juniperus phœnicea.

- thurifera.

Asphodeline lutea.

Stipa gigantea.

Echinaria capitata.

Avena bromoides.

Poa bulbosa.

Melica Cupani.

Festuca ovina var.

Ephedra nebrodensis.

Sur le versant ouest du Mahmel, nous trouvons, à une altifude de 2000 mètres, de beaux Blés encore en floraison, tandis que la moisson se fait dans la vallée.

Le 14 juillet est consacré à l'exploration des bois aux environs de la maison forestière de $\mathrm{Sgag}$, dans un site ravissant. Le Chêne vert et le Cèdre sont les essences dominantes; l'Acer monspessulanum, les Juniperus thurifera et J. Oxycedrus, Fraxinus dimorpha, Cotoneaster Fontanesii, complètent la végétation arborescente. Les plantes intéres- 
santes, encore en état, sont assez rares à cette saison; cependant nous notons :

Saponaria glutinosa.

Silene italica.

Onobrychis venosa.

Lonicera etrusca.

Galium verum (blanc et jaune).

Helichrysum lacteum.

Piptatherum paradoxum.
Avena macrostachya, Juncus caricinus. Poa flaccidula.

Festuca triflora.

- ovina.

- rubra.

Enfin un Podanthum qui nous paraît une espèce nouvelle et qui recevra le nom de Podanthum aurasiacum Batt. et Trab. La constatation de ce genre dans l'Aurès est l'indice d'une affinité avec l'Orient, tous les Podanthum connus de cette section habitant la partie orientale de l'Europe ou de l'Asie.

M. Franchet donne lecture d'une Note de M. Foucaud intitulée: Recherches sur quelques Enanthe (1).

M. G. Camus, secrétaire, donne lecture à la Société des communications suivantes :

\section{UN PEU DE DROIT A L'USAGE DES BOTANISTES HERBORISANTS,}

par M. Charles CORINEAU (2)).

Les herborisations et même les simples promenades sont tellement entravées, dans certaines contrées, par des propriétaires, ou plutôt par des gardes impitoyables, qu'il m'a paru intéressant d'étudier au point de vue technique le passage et la circulation sur la propriété d'autrui. On verra que le droit du propriétaire est beaucoup plus restreint, et celui du promeneur beaucoup plus large qu'on ne pourrait le croire, et je n'hésite même pas à dire que les propriétés sont, légalement et en droit strict, assez mal défendues.

Il est évident que l'on peut toujours, et malgré tous écriteaux prohibitifs, passer sur le terrain d'autrui, tant que ce terrain n'est pas clos et que l'on ne cause aucun dommage, et ce, alors même que les propriétaires ou les gardes chercheraient à entraver la circulation; c'est au propriétaire de se clore. Je ne eonnais à cette règle que de rares exceptions : je citerai d'abord les terrains de tir et de manœuvre, où l'interdiction n'existe que pour la sécurité du public; les bois soumis au régime forestier, dans lesquels on ne peut circuler en dehors des routes et chemins, et encore cette prohibition n'est-elle édictée

(1) Cette Note sera ultérieurement insérée, la planche qui doit l'accompagner n'étant pas terminée (Ern. M.).

(2) Notre confrère M. Copineau est juge au tribunal de Doullens. 


\section{$2 \mathrm{BHL}$ Biodiversity Heritage Library}

Trabut, Louis. 1892. "Herborisation Dans Le Massif De L'Aurès, Les 10, 11, 12 , 13, 14 Juillet." Bulletin de la Société botanique de France 39, 339-346. https://doi.org/10.1080/00378941.1892.10828673.

View This Item Online: https://www.biodiversitylibrary.org/item/8661

DOI: https://doi.org/10.1080/00378941.1892.10828673

Permalink: https://www.biodiversitylibrary.org/partpdf/159327

\section{Holding Institution}

Missouri Botanical Garden, Peter H. Raven Library

\section{Sponsored by}

Missouri Botanical Garden

\section{Copyright \& Reuse}

Copyright Status: Public domain. The BHL considers that this work is no longer under copyright protection.

This document was created from content at the Biodiversity Heritage Library, the world's largest open access digital library for biodiversity literature and archives. Visit BHL at https://www.biodiversitylibrary.org. 